\title{
INVESTIGACIÓN
}

Recibido: 04/05/2016 --- Aceptado: 17/10/2016 --- Publicado: 15/03/2017

\section{ANÁLISIS DE LAS REDES SOCIALES DE LAS MUJERES TRABAJADORAS. EL CASO DE LA LABOR DE INVESTIGACIÓN}

Gizelle Guadalupe Macías González: Universidad de Guadalajara. México ggmg261@yahoo.com

\section{RESUMEN}

El objetivo del documento es el de dejar ver la manera en que las redes de sociales son utilizadas por la mujer investigadora para lograr el quehacer investigativo. El estudio integra un análisis teórico sobre mujeres y trabajo partiendo desde las concepciones de la sociología del trabajo, valoradas desde la perspectiva de las mujeres, pretendiendo dar peso al posicionamiento del posterior auto relato centrado en mujeres que realizan investigación científica. Posteriormente se mencionan las concepciones de redes sociales y los análisis para abordarlas. El documento deja ver la relación del conocimiento situado, que parte de la propia investigadora con el tema en estudio sobre mujeres y trabajo, desde una tipificación de categorías identificadas como sus redes sociales del saber (de conocimiento), redes de colaboración profesional y redes de trabajo cotidiano. Analizando su frecuencia de uso, las escalas de confianza, las estructuras que se generan y las redes sociales que propician mayor enriquecimiento científico.

\section{PALABRAS CLAVE}

Redes sociales - Análisis de redes sociales - Mujeres - Trabajo - Mujeres trabajadoras - Mujeres investigadoras - Mujeres científicas.

The goal of the paper is to reveal how social networks are used by the woman researcher to achieve the research work. The study integrates a theoretical analysis on women and work starting from the conceptions of the sociology of the work, valued from the perspective of women, pretending to give weight to the positioning of the later self-story focused on women doing scientific research. Subsequently the conceptions of social networks are mentioned and the analyses to deal with them. The document reveals the relationship of located knowledge that starts from the researcher herself in the study theme on women and work, from a definition of categories identified as their social networks of learning (knowledge), networks of professional collaboration and networks of daily work. Analyzing the frequency of use, the scales of trust, the structures generated and the social networks that foster greater scientific enrichment.

${ }^{1}$ Gizelle Guadalupe Macías González: Doctora en Ciencias. Área: Ciencias Sociales y económicas. Líneas de investigación: Estudios de las mujeres en economía y educación, Educación y sociedad y, Economía, gestión de negocios y organización.

ggmg261@yahoo.com 


\section{KEY WORDS}

Social Networks - Social Networks Analysis - Women - Work - Women workers Women scientist - Women researchers.

\section{ANÁLISES DAS REDES SOCIAIS DAS MULHERES TRABALHADORAS. O CASO DO TRABALHO DE INVESTIGAÇÃO}

\section{RESUMO}

O objetivo do documento é deixar ver a maneira em que as redes sociais são utilizadas pela mulher investigadora para conseguir o trabalho de investigação. $\mathrm{O}$ estudo integra uma analises teórica sobre mulheres e trabalho desde as concepções da sociologia do trabalho, valoradas desde a perspectiva das mulheres, pretendendo dar importância ao posicionamento do posterior auto- relato centrado em mulheres que realizam investigação científica. Posteriormente, mencionam as concepções das redes sociais e as analises para abordar-las. O documento deixa ver a relação do conhecimento situado, que parte da própria investigadora com a questão em estudo sobre mulheres e trabalho, desde uma tipificação de categorias identificadas como suas redes sociais do saber (do conhecimento), redes de colaboração social e redes de trabalho cotidiano. Analisando sua freqüência de uso, as escalas de confiança, as estruturas que se geram e, as redes sociais que propiciam maior enriquecimento científico.

\section{PALAVRAS CHAVE}

Redes Sociais - Analises de Redes Sociais - Mulheres - Trabalho - Mulheres Trabalhadoras - Mulheres Investigadoras - Mulheres Cientistas.

\section{Cómo citar el artículo}

Macías González, G. (2017). Análisis de las redes sociales de las mujeres trabajadoras. El caso de la labor de investigación [Analysis of Social networks of women workers. The case of the work in research. Vivat Academia. Revista de Comunicación, 138, 56-76 doi: http://dx.doi.org/10.15178/va.2017.138.56-76

http://vivatacademia.net/index.php/vivat/article/view/1059

\section{INTRODUCCIÓN}

La intención de dar cuenta de las redes sociales que utilizan las mujeres investigadoras, por la labor de trabajo que realizan es la principal tarea del presente documento. Mostrar un análisis de redes sociales propuesto por distintos tipos de redes sociales de colaboración fue el camino que se pretendió seguir. Para ello convino partir por una parte, desde la consideración actual del trabajo que desempeñan las mujeres hasta llegar a la categoría específica de las mujeres investigadoras $\mathrm{y}$, por otra presentar y discutir las aproximaciones generales de las redes sociales -las redes personales, familiares, informales de intercambio, las redes de organización virtual, redes de colaboración científica y profesional y sus perspectivas de análisis-, tratando de ubicar casos específicos para las mujeres. 
Y como caso de oportunidad, se presentó la posibilidad de plasmar las reflexiones cotidianas de la autora en el campo de esta investigación. El objetivo del documento es el de dejar ver la manera en que las redes de colaboración son utilizadas por la investigadora para lograr el quehacer investigativo. A continuación se presenta el fundamento teórico partiendo desde las concepciones de la sociología del trabajo y del empleo, valoradas desde la perspectiva de las mujeres, con abordes feministas, pretendiendo dar peso al posicionamiento del posterior auto relato. El documento deja ver la relación del conocimiento situado, que parte de la propia investigadora con el tema en estudio (Blanco, 2012), desde una tipificación de categorías identificadas como sus redes del saber (de conocimiento), sus redes de colaboración profesional y sus redes de trabajo cotidiano-familiar.

Los aportes del estudio se realizan en el campo de la sociología del trabajo, desde una perspectiva feminista, visibilizando los ajustes y redes que la investigadora debe movilizar para continuar con su itinerario investigativo y potencializar su fortaleza profesional. El análisis se suma a los estudios cualitativos sobre experiencias de mujeres de la presente generación y su incorporación en el mundo del trabajo.

Las situaciones de trabajo pueden visualizarse con una nueva luz de análisis desde la sociología del empleo. La relación del empleo involucra una construcción social, al no solamente representar un fenómeno de mercado. Para Maruani (2000b) las recomposiciones de la población activa no son sólo movimientos demográficos o evoluciones económicas, son también procesos sociales que hallan sus raíces en la evolución de la sociedad y en los comportamientos de los actores sociales. Las definiciones de la socióloga francesa sobre trabajo y empleo, reside en conceptos más operativos, que conceptuales, más sociológicos que economicistas. En su postura conceptualiza y diferencia el trabajo (entendido como actividad de producción de bienes y servicios y conjunto de las condiciones de ejercicio de dicha actividad) del empleo (entendido como conjunto de las modalidades de acceso y salida del mercado de trabajo, así como la traducción de la actividad laboral en términos de estatus sociales), que aunque a primera vista, pareciera esquemático, la diferencia no carece de utilidad. El estatuto del empleo estructura el estatuto del trabajo, modifica la cartografía de las separaciones sociales en las empresas y organizaciones.

Por otra parte, los estudios del trabajo de los últimos años han mostrado una creciente incorporación laboral de la mayoría de los integrantes adultos de los hogares, propiciando nuevos procesos sociales en la organización familiar. Hombres y mujeres ingresan al mercado laboral y surgen adecuaciones familiares. En una organización de trabajo predominante, aparece una consideración más, dada por Abramo \& Todaro (2006) el trabajador normal y al anómalo, el primero se asocia con las rutinas cotidianas que son condicionadas por la asistencia laboral y el segundo se vincula con las rutinas caracterizadas de especificidades particulares. Las investigadoras mencionan que desde el ámbito laboral deben existir consideraciones de la vida extra-laboral de las personas, en el rediseño de los procesos de trabajo, políticas laborales y de recursos humanos. En la productividad social deben considerarse las necesidades de la vida familiar y personal, no solo cuestiones de reproducción biológica, sino requerimientos de formación cultural y ciudadana acorde con el desarrollo tecnológico, productivo y democrático. 


\subsection{El trabajo de las mujeres.}

En el análisis del trabajo y empleo, aparecen los avances sobre la perspectiva de las mujeres en el ámbito laboral. El enfoque de género en el mundo del trabajo es uno de los más evidentes en la sociedad, posiblemente por su trascendencia respecto al acceso a otros recursos sociales; el trabajo es uno de los espacios que más atención recibe en el esfuerzo colectivo por deconstruir la desigualdad de género. Hombres y mujeres tienen diferencias biológicas notorias y en el proceso de socialización se les atribuyen papeles y valores distintos; las desigualdades están socialmente construidas (Macías, 2013). La denominación de Cervantes sobre los consensos genéricos de las condiciones sociales de las mujeres, que menciona González (1997) son: que las mujeres comparten una situación opresiva en la sociedad (existiendo distintos niveles de análisis de lo social, al no percibir todas de la misma manera su condición y la existencia de diversas formas de desigualdad), y que las desigualdades sociales son edificadas.

Desde la década de los setentas hasta inicios del año dos mil Maruani (2000a) menciona que se han presentado diversas transformaciones, rupturas y brechas decisivas en la feminización del mercado de trabajo en un entorno de precariedad. Existiendo una afluencia en los avances de incorporación de mujeres al mercado de trabajo y al igual que los avances en la escolarización femenina. En el contexto europeo se analizan la historia del empleo femenino, sus variantes y la progresiva incorporación de las mujeres al trabajo, en entornos de crisis, de paros masivos, duraderos y estructurales y de incorporación caracterizada por una desigualdad basada en el sexo. Los primeros estudios sobre la actividad femenina se realizaron en los años sesentas y posteriormente en la década de los setentas aparecieron los movimientos feministas radicales y las incorporaciones de mujeres a universidades y a centros de investigación, como es el caso francés del Centre International de la Recherche Scientifique; donde se suscitaron estudios sobre el trabajo de las mujeres doméstico y profesional- , las relaciones de sexo y la división social del trabajo.

En esta misma perspectiva desde el entorno latinoamericano, Abramo \& Todaro (2006) enuncian que los costos de la reproducción y los domésticos son absorbidos por el trabajo no mercantil de las mujeres. No obstante generalmente las remuneraciones entre hombres y mujeres son distintas, para el panorama empresarial los costos de emplear a una mujer son mayores (por la asunción de responsabilidades domésticas y familiares, considerados factores adicionales a los costos, aunados al riesgo a la eventualidad), existiendo brechas de equidad de género relacionadas con las condiciones sociales de las mujeres. En este contexto aparece el cuestionamiento sobre ¿cuáles serían las medidas adecuadas para aumentar la productividad de las mujeres?, debido a que la productividad de trabajo de cualquier individuo, siempre depende del proceso y del contexto en el que se encuentran insertos. Las mediciones de actuales de productividad que citan las autoras integran una definición sistémica, que va más allá de un cálculo preciso de operaciones definidas en términos individuales al enunciar la postura de Zarifian dada en 1997. Volviéndose en el avanzar del tiempo con mayor complejidad (y a su vez insuficiente) al definir la 
productividad de cada individuo en particular o el aporte específico individual a la productividad global de la empresa (en su caso), sin considerar estos factores más sistémicos.

Sobre la ocupación de las mujeres por sectores, Abramo \& Valenzuela (2006) dan cuenta en su estudio sobre el empleo de hombres y mujeres realizado en 20 países latinoamericanos, de la incorporación de las mujeres en el sector servicios, como resultado de una posible absorción por expulsión de la industria o por subcontratación de servicios. Mencionan que el empleo femenino y el proceso de la terciarización, es más acentuado entre las mujeres, al encontrar en el sector servicios mayores posibilidades de trabajo, específicamente en comercio, en servicios comunales, personales y sociales.

\subsubsection{Mujeres investigadoras y cualificación profesional.}

La cualificación y el nivel profesional van vinculados al tipo de trabajo que se realiza. Maruani, (2000b) menciona cómo la cualificación se construye también, y sobre todo, partiendo de las condiciones y del estatuto del empleo que determinan la nomenclatura de las cualificaciones. La separación se establece entre empleos a tiempo parcial, estigmatizados de entrada como no cualificados, y empleos a tiempo completo que garantizan un mínimo de profesionalidad. En otras circunstancias, puede ser la duración del contrato laboral (por tiempo definido o indefinido) quien le da la jerarquía de las cualificaciones.

El valor del capital humano de las mujeres se relaciona con su cualificación o grado de preparación profesional. Daune-Richard (2000) menciona el caso francés presentado desde los años sesentas, donde el panorama del empleo de las mujeres se ha modificado. Ellas tanto más que los hombres se han beneficiado de la explosión de la formación escolar y universitaria de ese período, mostrando perfiles de actividad que les han permitido desarrollar su capital de experiencia, sin verse traducido en una mejora equivalente en igualdad de condiciones de los hombres. La relación de la formación y el empleo se mediatiza a través de la cualificación. A mayor nivel de formación existe acceso más amplio de empleos cualificados. En esta caracterización aparece la movilidad laboral femenina y los servicios. Maruani $\left(2000^{a}\right)$ afirmaba el acceso de muchas mujeres a las instituciones de investigación, sin embargo en el análisis del trabajo femenino mencionaba la acumulación de trabajos para las mujeres, los presentados entre las esferas familiar y profesional.

En el estudio realizado en México por Becerril \& López (1997) se mostraba que el sector servicios era el que mayor actividad de capacitación femenina mostraba. Y en la configuración de los rasgos del perfil de la mujer trabajadora mexicana resaltaban los criterios de sus niveles de instrucción y capacitación, relacionándose de forma significativa.

Sobre las mujeres que trabajan en México como investigadoras, y de acuerdo a los datos proporcionados por el Sistema Nacional de investigadores del CONACYT (2010), en su conformación se dejaba ver que una tercera parte estaba representado por las mujeres. Su participación mayor se encontraba en las áreas de humanidades y ciencias de la salud, y en los niveles de candidata a investigador nacional y, conforme 
avanzaba la jerarquía, su participación disminuía. El principal problema que se valoraba era que para ingresar, las mujeres deberían de contar con un trabajo.

\subsection{Redes sociales}

La temática de interés en estudio surge de dar cuenta de las redes sociales utilizadas por las mujeres en su trabajo, específicamente de las mujeres investigadorascientíficas. Para ello y después de revisar el panorama del trabajo de las mujeres, en un primer apartado se aborda el concepto de las redes sociales y posteriormente se realizan aproximaciones relacionadas a las redes personales y familiares, redes informales de intercambio, redes de organización virtual tanto como redes de colaboración científica y profesional, tratando de encontrar acotaciones relacionadas en específico con el caso de las mujeres.

Comenzando a indagar los conceptos, se detalla la aproximación realizada por Lozares (1996) sobre las redes sociales, mencionando que estas representan un conjunto bien delimitado de actores -individuos, grupos, organizaciones, comunidades, sociedades globales, etc., vinculados unos a otros a través de una relación o un conjunto de relaciones sociales. El autor menciona a Mitchell (1969) afirmando que las características de estos lazos en tanto que totalidad pueden ser usados para interpretar comportamientos sociales de las personas implicadas, pero parece más bien un objetivo genérico que un criterio especifico de definición. La identificación principal de las redes reside en valorarlas como unidades sociales que aparecen vinculadas unas a otras a través de diversas relaciones, donde se analiza los actores y sus lazos, díadas, tríadas, subgrupos y grupos. Establece el principio de cohesión, determinado por la fuerza causal de sus comportamientos, la intensidad de los lazos de comunicación, el cual marca también las fronteras del grupo. Incluye el principio de equivalencia, donde los actores equivalentes son los que poseen pautas de relación semejantes a los de su misma posición con relación a otros actores.

Dentro de la perspectiva de las redes sociales se conjuntan acción y estructura, interacciones sociales y normas institucionales, perspectivas micro y macro, esta perspectiva de acuerdo con Molina \& Aguilar (2004) puede ser de utilidad para la teoría social.

Por otra parte desde las tecnologías de la información y la comunicación, López (2014) rescata la definición de las redes sociales de Hernández al mencionarlas como las "asociaciones de personas ligadas por motivos heterogéneos y que conforman una estructura compuesta por nodos unidos entre ellos por más de un tipo de relación".

En esta visión tecnológica de redes y con el complemento de estudios de redes basados en la teoría social, se han profundizado recientemente estudios específicos de redes de colaboración científica.

\subsubsection{Redes personales, familiares y redes informales de intercambio.}

En la caracterización de las redes sociales de las mujeres, conviene rescatar las consideraciones de las redes personales, familiares y las informales. Para Molina (2005) en las redes personales se puede observar interacciones institucionalizadas (intercambios culturalmente dependientes de regalos entre familiares, de apoyo entre 
hijos adultos y sus padres, relaciones expresivas entre amigos, pero también relaciones cliente-proveedor, jefe-subordinado, político-elector) en el marco de estructuras sociales preexistentes (estratificadas por clase social y/o estatus, grupo ocupacional o residencial) que las influyen, condicionan o permiten. En el estudio de la vida social inmediata de las personas, se han presentado los estudios relativos a la comunidad, tradición de investigación que se centra en la localización de las redes de apoyo social, redes de iguales constituidas por parientes, amigos y vecinos que proporcionan socialización, información y ayuda en general.

En el esquema de estudio propuesto por Molina (2005) se incluye el análisis del tamaño de la red personal, la composición de los atributos de los alteri (relaciones de ego), los tipos de relaciones, el contenido de las relaciones, la estructura de la red personal y la dinámica de las redes personales. Menciona también la discrepancia fundamental que puede existir puesto que la memoria es selectiva y pudiera haber diferencias entre lo que la gente dice y lo que la gente hace. En sus resaltados obtuvo algunas consideraciones de las redes de las mujeres en cuanto al tamaño, percibiendo que la mujer suele estar subrepresentada en el mundo del trabajo formal y de la política, por lo que no es de extrañar que en promedio las redes sociales de las mujeres sean más reducidas. Por ello en tamaño las redes personales de las mujeres son en promedio, Menores. En cuanto a su composición, las mujeres tenderán a tener más mujeres en su red personal, es decir con sus iguales, debido a que las relaciones tienden a ser homófilas, por lo que el sexo, la clase social, la profesión y otras características consideradas relevantes en un contexto dado. En cuanto al contenido, las redes con más mujeres tienden a contener más apoyo social, mencionando que en la literatura suele distinguirse entre apoyo social (cotidiano y de emergencia), de socialización o confidencia y relaciones instrumentales, rescatando que otra vez el género es importante. $\mathrm{Y}$ en cuanto a su estructura da cuenta que seguramente las redes de las mujeres tienden a ser más densas por una variedad de razones (más frecuencia de contacto, más apoyo social, redes más pequeñas), la densidad de las redes personales en promedio es del $30 \%$.

Por otra parte la importancia de las redes familiares y de parentesco, son estudiadas por Adler Lomnitz (1994), identificando relaciones internas y externas y, relaciones preferenciales en contextos de igualdad de oportunidades. Ubicándolas en contextos no solo de personas con precariedades de vida, sino también en la constitución y estrategias de los grupos de -solamente- hombres de negocios. En su estudio sobre personas de escasos recursos, menciona que en la barriada, se presenta la asimetría incipiente en las relaciones internas a la red. $Y$ da cuenta que las redes de parentesco suelen ser la red social efectiva, la red de parientes, incluso el requerimiento de afiliación mencionándola como el compadrazgo.

Las redes informales de intercambio de Lomnitz (1990) se presentan cuanto más se formaliza, regula y planifica burocráticamente un sistema social que, pese a todo ello, no satisface las necesidades de la sociedad, y suelen crearse mecanismos informales que escapan al control del sistema. Estos modos informales de intercambio crecen en los intersticios del sistema formal, prosperan en sus ineficiencias y tienden a perpetuarlas al compensar sus defectos y al generar fracciones y grupos de interés dentro del sistema. La reciprocidad propicia el intercambio informal de bienes y servicios en un sistema social formal, surge en respuesta a la escasez, por ejemplo el 
intercambio en el mercado según los motivos, las metas, el grado de represión del intercambio o las reglas obligatorias culturalmente definidas entre las partes.

\subsubsection{Redes de organización virtual.}

Actualmente las tecnologías de la información y la comunicación están renovando todos los ámbitos de la vida humana, incluyendo por supuesto a las mujeres. La idea sobre el entorno como el espacio físico que rodea al ser humano ha sido modificado por el acceso al entorno virtual. Ortega \& Gacitúa (2008) afirman que ahora el entorno es construido en la red, al tener la oportunidad de ser, crear y participar en comunidades de interés personal, de producir información y reeditarla. Los procesos de mediación y comunicación en la perspectiva digital han conformado identidades y procesos específicos de aprendizaje, de construcción de conocimiento, de colaboración y de creación de comunidades. La conexión inmediata que caracteriza a las redes sociales han facilitado la creación natural de una inteligencia colectiva, con la inclusión de sistemas personales y a la vez diversos.

La consideración de las redes sociales no fue exprofeso para el uso educativo, Alvaréz (2014) parte de la teoría de los seis grados de separación, formulada por Frigyes Karinthy, una red social puede entenderse como "un sitio en la red cuya finalidad es permitir a los usuarios relacionarse, comunicarse, compartir contenido y crear comunidades",

En el ámbito científico el cambio está íntimamente relacionado con las redes sociales, tanto las de uso general como las redes sociales específicas de la ciencia. Ideadas como moda adolescente, se está convirtiendo en una herramienta revolucionaria de divulgación de resultados científicos -que cada vez son más en formato electrónico-. López (2014) valida que es imprescindible cambiar las rutinas y adaptarse a las tecnologías. Sin perder la continuación en grupos de personas con intereses comunes, trabajando en red dentro de un mismo ámbito de colaboración.

Los beneficios que manifiesta López (2014) derivan de la disolución de las fronteras espaciales e institucionales, ya que no es necesario estar vinculado a un colectivo para divulgar los estudios. Otro beneficio proviene del incremento de investigaciones son las colaboraciones de científicos de distintas latitudes y que perteneces a instituciones diferentes, algo tremendamente provechoso para la ciencia, por el tipo de colaboraciones que suele tener como resultado estudios brillantes.

Con cierta escasez, han aparecido algunos estudios de incorporación de las mujeres a estos espacios denominados redes, que manifiestan abordajes con ciertas particularidades como el que muestra Piñero (2014) sobre el texto denominado Madres en red, donde se da cuenta de la posibilidad que tienen las madres de informarse, expresarse y comunicarse on-line, permitiéndoles vivir etapas con un gran conocimiento y empoderamiento sobre los propios cuerpos y con la oportunidad de hacer visibles socialmente nuevos discursos entorno a la maternidad, hablando en primera persona sobre la experiencia personal y difundiéndola a grandes audiencias.

De igual manera y con base en el documento de Hernando \& Florian (2004) se potencializan las redes del saber de las personas, en una especie de autoinvestigación 
acción, con bastante potencialidad y fuerza que está transformando paradigmas académicos, en el desarrollo de la virtualidad en investigación autoformativa, en bibliotecas virtuales, conversaciones electrónicas y navegaciones por internet, prácticas de laboratorio (conversaciones en vivo -whats app), permitiendo desarrollar la calidad y pertinencia de la formación universitaria, profesional, y delas y los investigadores. Los autores afirman que la misma malla de internet amortigua los golpes del tradicionalismo educativo, al igual que la prudencia y conocimiento científico que aporta el saber o la habilidad para hacer las cosas, en una comunidad virtual de conocimiento, sin hacer distinción de género.

\subsubsection{Redes de colaboración científica y profesional.}

Actualmente se vive un crecimiento gradual de opciones de redes científicas. El documento de López (2014) da cuenta de redes formales denominadas: redes sociales específicas de la comunicación en la ciencia y para su estudio en el presente documento se consideran como redes informales, que son las comunidades científicas de intercambio de información que utilizan tecnologías participativas, asociadas algunas a redes sociales de uso común. En está sintonía las redes sociales de uso general son más colaborativas de lo que se pensó y la participación en estas redes es cada vez mayor. El autor menciona que Mallaparti encontró que "en los últimos cinco años ResearchGate, Mendeley y Academia.edu han generado individualmente una base de usuarios de casi dos millones de personas"

En el estudio de Henao (2012) para un investigador que utiliza las redes le puede representar un salto cualitativo en su investigación, al estar enriquecido con aportaciones de otros investigadores. Las alternativas de cambio de red científica se presentan cuando los científicos valoran la posibilidad de un incremento en su producción intelectual e investigativa y de acuerdo a la cita del autor sobre Bourdieu se estaría incrementando el capital cultural. La inclusión vendría acompañada de beneficios individuales y colectivos, al pertenecer a una red de investigadores y científicos implicaría efectos positivos en la producción investigativa al mejorar el desarrollo del trabajo científico o acrecentar la cantidad de los productos de investigación obtenidos. Las redes de colaboración científica tendrían un efecto multiplicador y a la vez generarían economías de escala, las cuales se constituyen en un incentivo positivo para los investigadores impulsándolos a cambiar de red geográfica, porque solo los científicos ubicados en ellas pueden beneficiarse de estas economías que son las que generan círculos intelectuales donde el autor subraya el término de clúster del conocimiento expuesto por Del Río. Las redes de colaboración científica y la red geográfica pueden presentar la fuga de cerebros. Henao (2012)en su caracterización también manifiesta que en los estudios de élite, las personas tienen más probabilidades de tener una conexión, relación o amistad si presentan similitudes o capital social, pudiendo representar un nodo élite en la red local, y con una posición privilegiada en la red global, convirtiéndose en un puente de entrada a la red del país desarrollado y a su red local, además de incrementar sus contactos. Las relaciones que se gestan entre los actores se perciben como fuentes de recursos tanto materiales como no materiales. 
Filippo, Sanz, \& Gómez (2007) valoran esa homogeneidad en particular y encuentran ese patrón de colaboración de vínculos nacionales e internacionales, de cercanía geográfica, idioma, vínculos históricos o socioeconómico donde resulta evidente que las relaciones se producen con centros de reconocido prestigio y calidad internacional; donde las publicaciones conjuntas son solamente uno de los productos de la colaboración, complementado al estudio de resultados y productos que pueden ser tanto tangibles como intangibles. Las estancias y las prácticas propias de publicación individual o colectiva, así como la difusión suelen tener especificidades por áreas de estudio. Para los autores la movilidad puede dar lugar a la formación de recursos humanos, creación de redes de intercambio, foros de debates y a la conformación de colegios invisibles, difícilmente cuantificables, por lo que valoran la utilizar metodologías no solo cuantitativas como las que utilizan encuestas, sino también la incorporación de entrevistas en profundidad para abordar problemáticas poco conocidas y difíciles de estudiar.

Para las mujeres en específico, el estudio de Arroyo, Bolaños, González, et. Al. (2010) muestra que en la colaboración científica las mujeres están presentes en todas las redes de al menos 10 autores y en algunos casos su presencia se equipara a la de los hombres. No obstante la participación de las mujeres en todas las áreas del conocimiento es inferior a los hombres, en las Ciencias de la Salud la proporción es menor que en otras áreas técnicas. En específico sobre las redes de autoría, destacan las redes compuestas por más de 20 autores ya que en su mayoría son hombres, y cuanto estás van disminuyendo, incluso se llegan a equiparar entre hombres y mujeres o incluso a tener un predominio de mujeres entre sus miembros.

Tratando de concretar la idea de este documento se ha percibido que al mencionarse redes sociales de las mujeres se estará haciendo referencia a la estructuras de intercambio social formales e informales suscitadas entre una persona o grupo de personas que se encuentren interrelacionadas y de acuerdo con López (2014) que incluso cubrirían las estructuras de intercambio de componentes sociales online

\subsection{Análisis de las redes sociales}

Se averiguaron distintos enfoques para realizar los análisis de las redes sociales, valorados desde diversas disciplinas como la antropológica, sociológica y desde las ciencias de la comunicación. De alguna manera se obtuvieron algunas particularidades específicas, pero difícilmente se obtuvieron acotaciones de análisis específicos desde el enfoque de las mujeres, salvo para el caso de las redes personales de Molina (2005), mencionado en apartados anteriores, donde aparecen especificidades susceptibles de examinarse desde la perspectiva femenina como el tamaño de la red personal, su composición, tipos, contenido, estructura y dinámica de relaciones personales establecidas. A continuación se mencionan de manera general cinco posibilidades, donde no aparecen acotaciones específicas de género. La propuesta de Sanz (2003). Valora que los científicos sociales utilizan conceptos y categorías relacionados con el análisis de redes con varias intenciones. Valida que el análisis de redes es una aproximación intelectual amplia para identificar las estructuras sociales que emergen de las diversas formas de relación, pero también un conjunto específico de métodos y técnicas. Relata la antigüedad de estos estudios, al 
mencionar que décadas atrás las tradiciones dominantes en las Ciencias Sociales han construido los modos de explicación científica. El análisis de redes sociales ARS (Social Network Analysis), también denominado análisis estructural, se ha desarrollado como herramienta de medición y análisis de las estructuras sociales que emergen de las relaciones entre actores sociales diversos (individuos, organizaciones, naciones, etc.). El análisis parte del estudio de la conducta individual a nivel micro y los patrones de relaciones (la estructura de la red) a nivel macro y las interacciones entre ambos niveles, pudiendo ser bilateral o multilateral, emergiendo una estructura denominada red social. La ARS ha sido consolidada con el apoyo de la álgebra matricial y de los grafos, que en ocasiones puede involucrar a varios conjuntos, donde se utilizan matrices de redes. La forma más directa de estudiar una estructura social es analizar los patrones de vínculos que ligan a sus miembros, buscando estructuras profundas. El autor menciona que la red es un constructo relacional, en el cual las descripciones se basan en los conceptos de vínculos (ties) que unen actores (nodes) que pueden ser personas, grupos, organizaciones o clusters de vínculos -así como de personas- en un sistema social. Los elementos de la red son los actores y las relaciones que se establecen entre ellos. Los primeros son representados por puntos en la red o nodos y los segundos por líneas. Si los actores se describen como nodos y sus relaciones como líneas entre pares de nodos, el concepto de red social pasa de ser una metáfora a una herramienta operativa analítica que utiliza el lenguaje matemático de la teoría de grafos, de las matrices y del álgebra relacional.

Aparecen las redes modo-uno donde todos los actores pertenecen a un único conjunto; el modo-dos, son las redes de afiliación, único conjunto de actores y de eventos. El análisis de la estructura general de la red y el nivel de integración grado de integración o la cohesión que la misma manifiesta. Análisis de la centralidad de los actores participantes en la misma. Interesa conocer la posición -posibilidades de poder de las mujeres-, grado, proximidad o cercanía y mediación. Ambas medidas de una red, asociadas al estudio de la centralidad y cohesión, tienen en cuenta, en lo fundamental: el número de organizaciones ligadas, el grado de exclusividad de los lazos y la posición de las organizaciones en el conjunto.

La intención de incluir en el estudio la especificidad del caso de las mujeres, permitiría ubicarlas en una centralidad.

La propuesta de Adler Lomnitz (1994) aborda análisis a profundidad y muestra en sus resultados entre otros modelos, diagramas de distancia social, de pareo o calce de favores con distancia social.

En los estudios de las redes sociales propuestos por Molina \& Aguilar (2004) se estudian las relaciones específicas entre una serie definida de elementos (personas, grupos, organizaciones, e incluso elementos del discurso). Distinguiéndose de los análisis tradicionales que explican, por ejemplo, la conducta en función de la clase social, la profesión o el grupo étnico; el análisis de redes sociales añade a los atributos las relaciones que existen entre los elementos, caracterizándose por contener datos relacionales o reticulares en el análisis. Un dato relacional representa un vínculo específico existente entre un par de elementos y a partir de los pares de elementos y la relaciones establecidas entre ellos es posible construir una red, pudiendo representar fenómenos muy diversos. Y desde el análisis de las redes personales, Molina (2005) bosqueja varias experiencias, estudios y metodologías de aproximación 
formulando estimaciones, figuras de jerarquía de estructura y de redes, entre otras herramientas.

Henao (2012) por su parte demuestra que los vínculos entre los diferentes actores sociales pueden indicar, primero, transferencias de recursos tanto materiales como no materiales, y segundo, la estructura social o económica que da sentido a las relaciones y permite mirarlas como relaciones que ofrecen oportunidades así como que restringen la acción individual.

\section{OBJETIVO}

El objetivo que el documento persigue es demostrar la manera en que las redes sociales son utilizadas por las mujeres trabajadoras para lograr el quehacer investigativo.

Tratando de conjuntar, por un lado, la experiencia personal femenina basada en los arreglos cotidianos establecidos con las redes sociales, vislumbrando la complejidad de realizar las labores asociadas a la condición social de las mujeres y por otro, de lograr efectuar el trabajo como investigadora social. Testimonio realizado y analizado desde la posibilidad de la construcción de la memoria social, a través del autorrelato. Dando cuenta del cómo se llega a lograr el objetivo laboral en el quehacer de la investigación científica, como parte de la vida femenina; en una situación en la que puedan reconocerse otras mujeres de generaciones similares y/o espacios geográficos heterogéneos. En cierto punto, un tanto afín a lo dicho por las propias experiencias como mujeres investigadoras que plantearon Villegas \& Mendoza (2015), tratando de manifestar la comprensión y configuración de procesos intergeneracionales de las mujeres científicas a través de discursos narrativos y en este caso desde la inclusión de las redes sociales.

\section{METODOLOGÍA}

El propósito de las ciencias sociales es la comprensión; se conceptualiza la lógica y se detallan los límites de su propia explicación, reconociendo la complejidad de lo real más que una generalización, reconocida desde el enfoque cualitativo en casos individuales (Della Porta y Keating, 2013). La historia del presente representa para Soto (2004) y Blanco (2011) la posibilidad de analizar un tránsito en la realidad social vigente, la historia vivida y su escritura en un registro histórico de lo que vive la persona, dando cuenta de contextos, leyendo una sociedad a través de una biografía de cursos de vida en trayectorias profesionales. Que para Blanco (2011) constituye una investigación científica, distinguiendo la ficción de la verdad, poniendo mayor énfasis a la faceta personal, al ámbito cultural o al propio proceso de investigación. Por su parte Cruz, Reyes y Cornejo (2012) consideran a los autorrelatos como datos de la investigación, reconociendo un conocimiento situado, en el que se participa al ser parte de la generación, representando el objeto y sujeto, activo y dependiente; asumiendo no solo un ejercicio epistemológico, sino también ético al otorgar mayor visibilidad a las cuestiones del propio punto de vista del tema de investigación

Con el anterior fundamento, la investigación que se lleva a cabo, deriva de una metodología que por una parte requirió de un análisis teórico sobre el trabajo de las 
mujeres y de las redes sociales, y por otra de un análisis empírico basado en la narración propia. Haciendo uso de la acción interpretativa y cualitativa de la experiencia personal como investigadora, en cuanto a las redes sociales utilizadas, valorándose desde el contexto del trabajo. Se utiliza la técnica del autorrelato para narrar el ¿cómo se asume de manera individual el reto de activar las redes sociales de colaboración femenina para cumplir con el cometido del trabajo científico?

Posteriormente se realizan ciertos análisis de las redes sociales que fueron elegidos del abordaje que se realizó en párrafos anteriores y desde el autorrelato mediante pautas de producción e informes del caso, señalando la ruta de vida predefinida por el objeto de estudio que en este caso representan las redes de colaboración de las mujeres frente al trabajo remunerado, visualizado en la labor de la investigación. Cruz, Reyes y Cornejo (2012) proponen averiguar: ¿cuál es el clima/tono del autorrelato?, ¿cuál es la trama y/o intriga que lo articula?, ¿qué hechos, hitos y/o escenas emergen como relevantes?, ¿cuáles son los personajes de la trama/intriga?, ¿desde qué lugar/posición social se narra el autorrelato?, ¿qué pistas hay sobre cómo aparece la dialogía?, pero también como dispositivo que facilitaría la producción de los datos, es decir, la escucha hacia los relatos de otros: ¿qué aporta el autorrelato para la empatía con el entrevistado/a; para la comprensión del contenido de los relatos de vida; para el posicionamiento de la investigadora en la dialogía intergeneracional; para la constitución del equipo de investigación?

\section{RESULTADOS}

Por cuestiones de espacio en este documento trato de abstraer la información que valoro sea narrada en mis redes sociales de colaboración. Recordando un primer ejercicio testimonial que realicé el año pasado me di cuenta que describía en un documento que "a mi parecer era gratificante participar activa y cotidianamente en la formación integral de los hijos, de recursos humanos y el compartir con los otros. Pero definitivamente no se tiene la estructura de cubrir y partir de las mismas condiciones de igualdad entre investigadoras e investigadores, sin responsabilidades familiares cotidianas, representando fronteras de las mujeres en sus hogares y en su desarrollo profesional como la investigación" (Macías, 2015)

Parto de esta reflexión para mencionar las redes sociales que están presentes en mi cotidianeidad de trabajo investigativo. Naturalmente estructuro mi narración identificando tres tipos de redes que visualizo. Las primeras son las redes del saber, donde trato de identificar como investigadora las oportunidades de conocimiento, de conocer que establezco a través de este tipo de redes sociales. Las segundas son las redes de colaboración profesional, donde me refiero singularmente a las redes sociales de mi entorno institucional cotidiano. Las últimas son las que denomino redes de trabajo cotidiano, refiriéndome a las relaciones de apoyo que establezco para llevar a cabo responsabilidades familiares y domésticas. Siempre considero que las he establecido de manera formal e informal y presencial o virtual. 


\subsection{Autorrelato de mis redes.}

a) Redes del saber

Recuerdo que desde cuando comienzo a realizar la labor de la investigación con un enfoque profesional -un poco desde mis estudios de doctorado y posteriormente con la obtención del grado-, me doy cuenta que para coincidir, discutir y compartir, he necesitado naturalmente buscar, establecer o encontrar colegas pares interesados en similares áreas de estudio y enfoques.

Mis primeras redes del saber, de conocimiento paralelo, surgen al asistir a eventos de participación y agrupación académica. Los congresos, coloquios, seminarios, foros y cátedras me representaron oportunidades de intercambio de saberes, metodologías, enfoques y abordajes de temáticas y objetos de estudios comunes. Desde mi formación doctoral autogenere la oportunidad de participar en un foro de género en la Universidad Pedagógica Nacional, registrando mi asistencia a través de mi primera ponencia, desean discutir con iguales mis ideas. Desde ese momento comienzo a presentar mis posturas y ubico regularmente algunos pares, generalmente mujeres-, con quienes intercambio cuestionamientos e inquietudes de abordar problemáticas de varios tipos, que surgen en una primera charla y al menos ubico nombre o correo electrónico. La mayoría de los eventos de investigación a los que asisto son en universidades distintas al CUALTOS de la UdeG donde laboro. Con las investigadoras -y en ocasiones con estudiantes de posgrado- que tengo mayor afinidad y que establezco la empatía, después de un primer acercamiento físico o virtual, o con las que ellas me recomiendan, trato de no perder el contacto y establezco medios para continuar con ese seguimiento, últimamente no solo el correo electrónico sino también la inclusión de relación mediante comunidades como academia.edu, facebook y whatsapp, caracterizadas por un acompañamiento cauteloso de largo alcance en tiempo. En ocasiones me comunico abriendo posibilidades por conjeturas que se me presentan, como dictaminación de obras o invitación a congresos en general o a simposios que coordino o seguimiento de información como memorias que algún día tuvimos en común y de manera muy particular -con algunas en específico- con mayor profundidad seguimientos a proyectos de investigación. A ciertas colegas con las que he trabajado o con las que solamente las he leído y me agrada su temática no las conozco físicamente; con algunas he superado un único encuentro vía correo electrónico y he establecido otros vínculos electrónicos (buscando sus fotografías, divulgaciones y difusiones en revistas, universidades, asociaciones, google, facebook, y recientemente en comunidades científicas de la red y whatsapp. Uno y otro proyecto a los que las invito y otros de los que recibo su participación. Con quienes tengo mayor reciprocidad cotidiana -virtual y en ocasiones física- son con dos investigadoras del centro del país. Aunque ahora me encuentro con una situación especial, ya dos de ellas ya están jubiladas. Y según los proyectos la reciprocidad virtual se acrecienta o disminuye en ciertos periodos con investigadoras nacionales e internacionales en contextos de colaboración. Estas redes sobre estudios de las mujeres en su mayoría han superado la barrera de la vinculación formal mediante convenios exprofesos. Y de manera formal he adquirido membresías de asociaciones para mantenerme al margen de sus avances y propuestas, una por recomendación y la otra por que la he valorado. 
b) Redes de colaboración profesional

De manera formal pertenezco a un cuerpo académico en mi institución a la par de otros colegas, colaborando en proyectos comunes y generalmente de iniciativa colaborativa con el tema educativo en común, solamente que yo lo desarrollo de manera secundaria. Por afiliación personal, he trabajado -no muy comúnmente- con investigadores de mi universidad en proyectos que nos vinculan. Y también por cuestiones de cohesión, reciprocidad y buena voluntad establezco relaciones de apoyo en procedimientos administrativos y académicos con pocos compañeros de mi institución, de algunos he recibido esporádicamente acompañamiento profesional que han hecho que supere mi campo de actuación o de los cuales me he apoyado en cuestiones de cubrir necesidades del ser humano. La comunicación que prevalece es la derivada del contacto físico cotidiano, que se confirma con la vinculación electrónica similar a las utilizadas en las redes del saber con objetivos de corto plazo.

c) Redes de trabajo cotidiano

En definitiva esta última agrupación proviene desde la valoración de la perspectiva de género, los estereotipos y roles sociales atribuidos a las mujeres y a la familia e incluye rutinas donde se suman las amistades. Trabajo que desempeño y de los que me apoyo en una dinámica familiar y doméstica. Con mi esposo preparo alimentos para distintos tiempos del día y con los hijos se delegan algunas actividades, con la característica de ser más de instrucción. Las labores de limpieza del y el cuidado de un par de horas de algún hijo se realizan por un vínculo con una persona de confianza que se le retribuye. Al igual que la labor de traslados y alimentación de los hijos son realizados en su mayoría por mi esposo, o esporádicamente por una de mis hermanas, mi tía o mi padre. Algunos tiempos de cuidado de algún hijo, por realizar labores investigativas o académicas externas a las tardes compartidas conmigo o en ocasiones con su papa, se presentan generalmente con la familia, con una de mis hermanas, con la familia de un hermano de mi padre, la madre de mi marido o esporádicamente con mis padres. Las gestiones para la obtención de provisiones que realizo en mi hogar son apoyadas en ocasiones por mis padres. El contacto con otros familiares y amigos se presenta de manera cotidiana pero raramente se les solicita ayuda, aunque últimamente se ha pedido el apoyo investigativo a las amistades, por cuestiones del objeto de investigación y de apoyo en trabajo de campo y con los sujetos. Salvo el caso de una hermana de mi madre y el esposo de mi primera hermana con los que acudo para apoyos de solventar provisiones. Últimamente recurro de manera virtual con mi segunda hermana y su esposo para apoyos médicos y recomendaciones saludables a la par de mi padre. El alojamiento en poblaciones cercanas distintas a la que resido me es brindado por mi segunda hermana y por la hermana de mi madre. Las opciones de traslados geográficos de tipo investigativo se ven apoyadas en las redes sociales de trabajo cotidiano con mi padre o marido o con la red social de colaboración profesional con compañeras académicas. 


\subsection{Análisis}

A continuación se presentan unos primeros análisis de las redes sociales de mi experiencia en investigación a través de algunas matrices y figuras. Las frecuencia de sus usos se presentan en la tabla 1, y en la figura 1 muestro la estructura que me proporciona mayor crecimiento científico. En la tabla 2 enlisto de qué redes sociales me valgo para realizar mi quehacer investigativo y en la figura 2 elaboro un diagrama de pareo o calce de favores relacionados con mi labor de investigación científica.

Tabla 1: Frecuencia de usos de las redes sociales.

\begin{tabular}{|l|c|c|c|c|}
\hline \multicolumn{1}{|c|}{ Tipos de redes sociales } & Físicas & Virtuales & Informales & Formales \\
\hline Redes del saber & 2 & 5 & 5 & 2 \\
\hline $\begin{array}{l}\text { Redes de colaboración } \\
\text { profesional }\end{array}$ & 3 & 3 & 2 & 2 \\
\hline Redes del trabajo cotidiano & 5 & 5 & 5 & 1 \\
\hline
\end{tabular}

Escala de utilidad asignada:

5. Se utilizan en su totalidad, 4. Se utilizan casi en su totalidad, 3. Se utilizan algunas veces, 2 . Casi no se utilizan y 1 . No se utilizan

Fuente: Análisis del autorrelato

Figura 1: Estructura de las redes sociales de la mujer investigadora, propiciadora de mayor crecimiento científico.

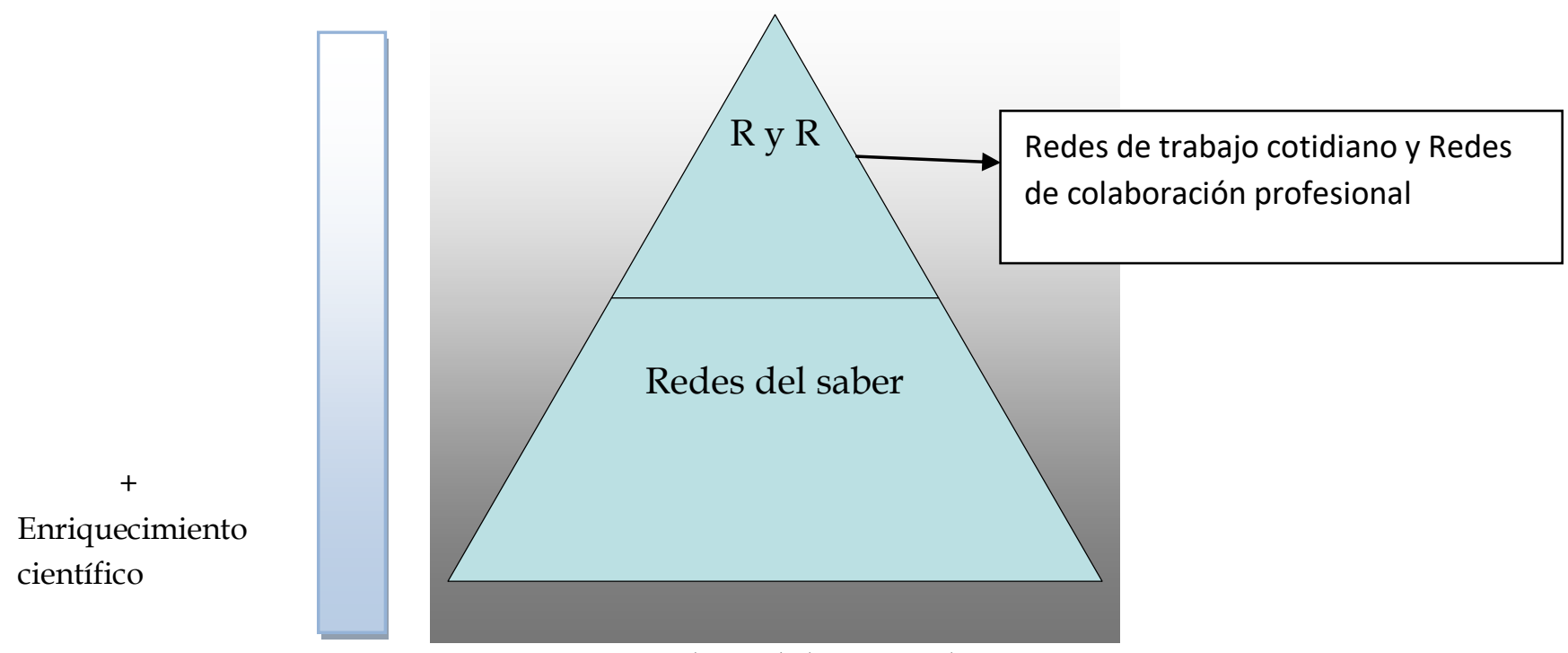

Fuente: Análisis del autorrelato 
Macías González, G. Análisis de las redes sociales de las mujeres trabajadoras. El caso de la labor de investigación

Tabla 2: Redes sociales utilizadas para el quehacer investigativo.

\begin{tabular}{|l|c|c|c|}
\hline \multirow{2}{*}{$\begin{array}{c}\text { Actividades necesarias para realizar el trabajo } \\
\text { investigativo }\end{array}$} & Redes sociales en las que me apoyo \\
\cline { 2 - 4 } & Redes del saber & $\begin{array}{c}\text { Redes de } \\
\text { colaboración } \\
\text { profesional }\end{array}$ & $\begin{array}{c}\text { Redes del } \\
\text { trabajo } \\
\text { cotidiano }\end{array}$ \\
\hline $\begin{array}{l}\text { Acrecentar mi conocimiento de mis líneas de } \\
\text { investigación }\end{array}$ & Sí & No & No \\
\hline Obtención de productos tangibles de investigación & Sí & Sí & Sí \\
\hline $\begin{array}{l}\text { Obtención de productos intangibles para la } \\
\text { investigación }\end{array}$ & Sí & Sí & Sí \\
\hline Traslado a eventos de investigación y académicos & No & Sí & Sí \\
\hline Recomendaciones del trabajo de investigación & Sí & No & No \\
\hline Dictaminaciones de trabajos & Sí & No & No \\
\hline Formación de recursos humanos & Sí & Sí & Sí \\
\hline $\begin{array}{l}\text { Apoyos para participación en eventos de } \\
\text { investigación }\end{array}$ & Sí & Sí & Sí \\
\hline Colaboración conjunta en investigación & Sí & No & Sí \\
\hline $\begin{array}{l}\text { Apoyos familiares para realizar labores de } \\
\text { investigación }\end{array}$ & No & No & No \\
\hline $\begin{array}{l}\text { Conciliaciones de tiempo para dedicar a la } \\
\text { producción académica }\end{array}$ & &
\end{tabular}

Fuente: Análisis del autorrelato

Figura 1: Diagrama de pareo o calce de favores investigativos con la distancia social o confianza.

Escalas de favores investigativos (virtual, físico, tangible, intangible)

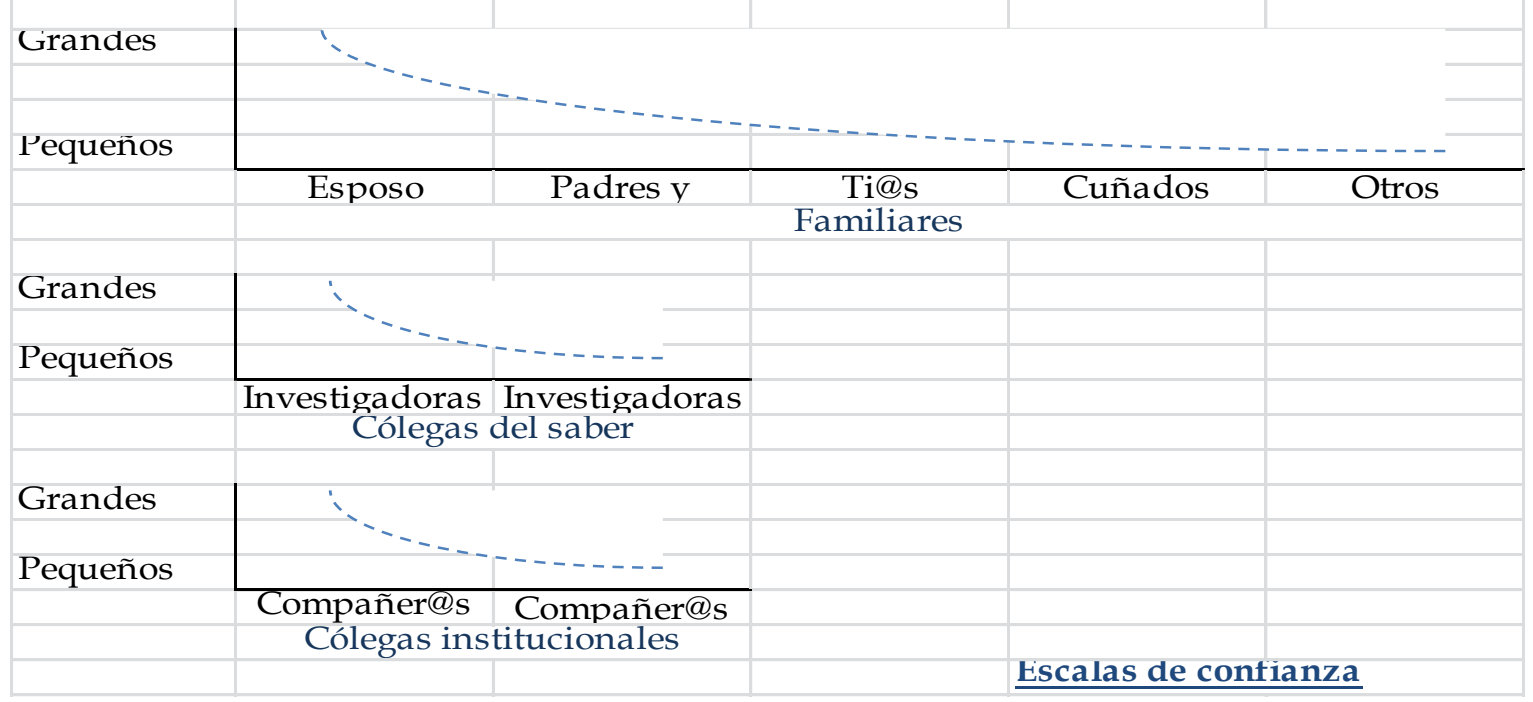

Fuente: Análisis del autorrelato, basado en Adler-Lomnitz (1994)

\section{DISCUSIÓN}

La oportunidad de mostrar la trayectoria personal y de no silenciar la voz y reconocer el por qué me apasiona, me causa conflictos y sensaciones; llevándome a tomar conciencia de lo enunciado por Legrand citado por Cruz, Reyes y Cornejo (2012) sobre la contratransferencia de la investigadora. Ahora desde un 
reposicionamiento personal me permití distanciarme del pasado, al relatarlo y acercarme al presente a partir de esta nueva lectura.

El fundamento teórico abordado dio pie a caracterizar el trabajo de las mujeres contextualizado actualmente, a través de un enfoque feminista y de comparación donde la condición social se asume ampliamente. Para realizar el enfoque de las mujeres investigadoras se documentó el creciente aumento de su presencia en estas áreas de estudios y posteriormente se mostraron las concepciones de las redes sociales, las redes personales, familiares y las informales de intercambio, las que han aparecido últimamente con la utilización de las tecnologías y las de colaboración científica y profesional conformadas en la actualidad. A manera de tener una evidencia más empírica y aprovechando la oportunidad se mostró con la narración personal, la manera en que las redes sociales son utilizadas por las mujeres trabajadoras para lograr el quehacer investigativo.

El autorrelato interpretativo muestra desde el enfoque de las mujeres el conocimiento situado en la investigadora. Se visualiza el reto de movilizar las redes de colaboración del contexto laboral y social para poder continuar en el itinerario de trabajo elegido, la labor de la investigación. La superioridad de las labores asignadas de la condición de ser mujer se mantiene con prioridad a la labor de realizar la investigación, donde los arreglos con las redes sociales juegan un papel primordial para posibilitar y dejar hacer el quehacer investigativo.

La frecuencia continua de las redes del saber y las redes del trabajo cotidiano establecidas de manera física, virtual e informal impactan de manera directa en el éxito de los estudios de la investigadora.

El mayor acrecentamiento científico de la investigadora que se determina la estructura piramidal proviene y es propiciado de manera contundente por las redes que establece sobre el saber, la visión paralela, el acercamiento a investigadoras de gran calidad científica y/o humana de nivel nacional e internacional ha permitido escalar y lograr los cometidos valorados por los indicadores de investigación. Y complementando lo evidenciado por la matriz todas las actividades necesarias para realizar el trabajo investigativo tiene su impacto en estas redes, salvo el traslado a los eventos de investigación y dos más que derivan de la conciliación familiar-doméstica. De alguna manera las otras dos redes de colaboración profesional y de trabajo cotidiano -que involucra familiares y amistades- tienen cierta injerencia de menor peso. En un análisis a detalle de los actores de las redes sociales que se utilizan para llevar a cabo la investigación deja ver que mientras el nivel de confianza en ellos aumenta, se acrecienta la dimensión del favor solicitado respecto a la investigación.

Los aspectos recogidos en este estudio pueden ser aplicables a varias áreas del conocimiento, y como se aprecia en los resultados obtenidos, existe una preocupación por la participación de la mujer en el contexto científico y el rol que se tiene con el fin de lograr el objetivo y alcanzar una paridad entre hombres y mujeres, en las categorías profesionales superiores citadas por Arroyo, Bolaños, González, et. Al. (2010). Una futura investigación derivaría en un enfoque netamente comparativo con la experiencia de un autorrelato y un análisis de las redes sociales de los hombres investigadores, tratando de visualizar y dejar en claro semejanzas y diferencias. De igual manera una evaluación de mayor detalle con instrumentos específicos de 
Macías González, G. Análisis de las redes sociales de las mujeres trabajadoras. El caso de la labor de investigación

análisis de redes sociales con enfoques comunicativos, antropológicos y sociológicos que requerirían mayor profundidad.

\section{REFERENCIAS}

Abramo, L. \& Todaro, R. (2006) Costos laborales y reproducción social en América Latina. En L. Abramo, (Ed.) Trabajo decente y equidad de género en América Latina. (pp. 129-152). Santiago, Chile: Oficina Internacional del Trabajo

Abramo, L. \& Valenzuela, M. E. (2006). Inserción laboral y brechas de equidad de género en América Latina. En L. Abramo, (Ed.) Trabajo decente y equidad de género en América Latina (pp. 29-62) Santiago, Chile: Oficina Internacional del Trabajo

Adler-Lomnitz, L. (1994). Redes sociales, cultura y poder. Ensayos de antropología latinoamericana. México: Miguel Ángel Porrúa.

Aguado, L. \& Rogel, R. (2002) La recuperación del observador en la construcción del dato. Cinta de Moebio. Revista de Epistemología de Ciencias Sociales, 13, 2-20

Arroyo, A., Bolaños, M., González, G., Villamon, M. \& Aleixandre, R. et. Al. (2010). Análisis de género, productividad científica y colaboración de las profesoras universitarias de Ciencias de la Salud en la Comunidad Valenciana (2003-2007). Revista Española de Documentación Científica, 33(4), 624-642.

Becerril, L. \& López, M. de J. (1997). La mujer trabajadora: sus condiciones de instrucción y capacitación. En M. L. González, (coord.), Mitos y realidades del mundo laboral y familiar de las mujeres mexicanas. México, D.F.: Siglo XXI editores e IIEC, UNAM

Blanco, M. (2012) ¿Autobiografía o autoetnografía? Desacatos, núm. 38, 169-178

Cruz, M.A., Reyes, M. J. \& Cornejo, M. (2012) Conocimiento situado y el problema de la subjetividad del investigador/a. Cinta de Moebio. Revista de Epistemología de Ciencias Sociales, $45 . \quad$ Recuperado de: http:/ / www.facso.uchile.cl/publicaciones/moebio/45/cruz.html

Daune-Richard, A. M. (2000) III. Cualificación y representación social. En M. Maruani, C. Rogerat \& T. Torns, (Dirs.) M. Bofill, (Trad.) Las nuevas fronteras de la desidgualdad. Hombres y mujeres en el mercado del trabajo. (pp. 71-86) Barcelona: Icaria Antrazy

De Filippo, D., Sanz, E. \& Gómez, I. (2007) Movilidad de investigadores y producción en coautoría para el estudio de la colaboración científica. Revista CT, 8(3), 23-40.

Della Porta, D. \& Keating, M. (2013). Comparación entre enfoques, metodologías y 
métodos. Conclusiones finales. En D. Della Porta, \& M. Keating, (Eds.) R. Vázquez, (Trd.) Enfoques y metodologías de las ciencias sociales. Una perspectiva pluralista (pp. 333-339) Madrid: Ediciones Akal

Duero, D. (2006) Relato autobiográfico e interpretación: una concepción narrativa de la identidad personal. Athenea Digital, 9, 131-151.

González, M. (1997). Mujer, fecundidad y trabajo. En González, M. L. (coord.), Mitos y realidades del mundo laboral y familiar de las mujeres mexicanas. México, D.F.: Siglo XXI editores e IIEC, UNAM

Henao, Diana Carolina (2012). La teoría de redes: aspectos claves para el análisis de la fuga de cerebros. Trans-pasando Fronteras, 2, 97-109.

Hernando, J. \& Florian, M. (2004). Redes del saber: investigación virtual, proceso educativo y autoformación integral. Bogotá: Magisterio.

INMUJERES (2011). Sólo una tercera parte del sistema nacional de investigadores son mujeres. México: Instituto Nacional de las Mujeres.

Lomnitz, L. (1990) Redes informales de intercambio en sistemas formales: un modelo teórico. Comercio exterior, 40(3), 212-220.

López, C. (2014) La interrelación entre las redes sociales específicas de la comunicación científica y las redes sociales de uso general. Vivat Academia. Revista de Comunicación, 127, 103-116. doi:doi.org/10.15178/va.2014.127.103-116

Lozares C., (1996). La teoría de redes sociales. Papers, 48, 103-126.

Macías, G. (2013) Introducción. La situación de las mujeres frente al trabajo y su participación social. En G. Macías, y E. Parada, (Coord.). Mujeres, su participación económica en la sociedad. Guadalajara, México: Editorial Universitaria de la Universidad de Guadalajara, Instituto Jalisciense de las Mujeres.

Macías, G. (2015) Experiencia femenina en la investigación y difusión científica. En A. Mendieta. ¿Legitimidad y reconocimiento? Las investigadoras del SIN, Retos y propuestas. Puebla, México: Benémerita Universidad Autónoma de Puebla. Consejo Nacional de Ciencia y Tecnología (CONACYT).

Maruani, M. (2000a) Introducción. En M. Maruani, C. Rogerat, \& T. Torns, (Dirs.) M. Bofill, (Trad.) Las nuevas fronteras de la desigualdad. Hombres y mujeres en el mercado del trabajo (pp. 15-25) Barcelona: Icaria Antrazy.

Maruani, M. (2000b) De la sociología del trabajo a la sociología del empleo. Política y Sociedad, 34, 9-17. 
Macías González, G. Análisis de las redes sociales de las mujeres trabajadoras. El caso de la labor de investigación

Molina, J. L. (2005) El estudio de las redes personales: contribuciones, métodos y perspectivas. Revista Empírica, 10, 71-106.

Molina, J. L. \& Aguilar, C. (2004) Redes sociales y antropología: un estudio de caso (discursos étnicos y redes personales entre jóvenes en Sarajevo). En: C. Larrea, \& F. Estrada. Antropología en un mundo en transformación. Barcelona: Editorial Icária.

Ortega, S. \& Garcitùa, J. C. (2008). Espacios interactivos de comunicación y aprendizaje. La construcción de identidades. RUSC. Revista de Universidad y Sociedad del conocimiento, 5(2).

Piñero, T. (2014). Madres en red. Del lavadero a la blogosfera. Vivat Academica. Revista de Comunicación, 128, 128-129. doi: https://doi.org/10.15178/va.2014.128.128-129

Sanz, L. (2003). Análisis de redes sociales: o cómo representar las estructuras sociales suyacentes. Apuntes de Ciencia y Tecnología, 7.

Soto, A. (2004) Historia del presente: Estado de la cuestión y conceptualización. Historia Actual Online, 3, 101-116.

Villegas, I. \& Mendoza, G. (2015) Como ser mujer en el SNI y no morir en el intento. En A, Mendieta. ¿Legitimidad y reconocimiento? Las investigadoras del SIN, Retos y propuestas. Puebla, México: Benémerita Universidad Autónoma de Puebla. Consejo Nacional de Ciencia y Tecnología (CONACYT).

\section{AUTORA}

\section{Gizelle Guadalupe Macías González}

Departamento de Estudios Organizacionales, Universidad de Guadalajara, Centro Universitario de los Altos. Profesora investigadora de tiempo completo, Perfil PROMEP, miembro del SNI, nivel Candidato. Integrante del Cuerpo Académico Educación y Sociedad, CA-UDG-433. Sus líneas de investigación: Estudios de las mujeres en economía y educación. Educación y sociedad. Economía, gestión de negocios y organización. Doctora en Ciencias, por la Universidad de Guadalajara Publicaciones: Sobre mujeres empresarias, mujeres y empleo, educación superior, emprendimiento, género, microfinanzas y estudios económicos regionales. Docencia en Microeconomía, Finanzas municipales y Desarrollo de emprendedores. Participante de la Red de Organizaciones, Desarrollo y Género de la UAA, UASLP, UGto., USMICH, UdeG. Miembro de la Academia Mexicana de Ciencias Administrativas, y de la International Association for Feminist Economics. https://guadalajara.academia.edu/GizelleMac\%C3\%ADasGonz\%C3\%A1lez 